Reprod. Immunol. Biol. 20(1): 10-12.

JSIR Sympodium

\title{
Genes on the autosomal chromosome related to gametogenesis
}

\author{
Naoki Takeshita* and Harumi Kubo \\ Department of Obstetrics and Gynecology, School of Medicine TOHO University. \\ 6-11-1 Ohmori-Nishi Ohta-Ku, Tokyo 143-8541 \\ *E-mail:ntakesit@med.toho-u.ac.jp
}

\section{Background}

Deletion of the Yq11 region of the $Y$ chromosome long-arm in male subjects with azoospermia was first reported in 1976, as discovered in a study conducted to explore the relationship between human azoospermia and the $Y$ chromosome. This region was named Azoospermic Factor (AZF) [1]. Many investiga-tors have subsequently pursued strenuous studies aimed at analyses of the $Y$ chromosome and the process of spermatogenesis. As a result, several genes have been identified and proposed as spermatogenesis-related genes, such as USP9Y, $D B Y, R B M Y$, and $D A Z$ (deleted azoospermia) [2].

Occurrence of microdeletion in the $A Z F$ region with significantly high frequency in infertile male subjects was reported in about 1996 and thereafter [3]. The success of intracytoplasmic sperm injection (ICSI) technique by Palermo G.D. et al. in 1992 [4] has brought about a substantial change in the conventional treatment for male infertility, and has been a great boon to such patients. In the meantime, nevertheless, it has been learned that microdeletion in the $Y$ chromosome might be transmitted via the assisted reproductive technology (ART) represented by ICSI. However, correlation between clinical manifestations and the deletion has not yet been established. Recently, a report demonstrating that a deleted azoospermia-like autosomal (DAZLA) gene, highly homologous to the $D A Z$ gene, is localized on the chromosome; $3 \mathrm{p} 24$, and has been attracting attention as to its relation with spermatogenesis [5]. The occurrence of BOULE, highly homologous to the $D A Z$ gene on chromosome 2, has also been reported. It has also been documented that the SYCP3 gene localized on chromosome 12 has a bearing upon meiosis in the spermatogenic process [5]. Thus, occurrence of genes regulating spermatogenesis not only on the sex-chromosome but also on autosomal chromosome has become generally recognized. DAZLA and BOULE genes are expressed not only in spermatids but in oocytes, probably taking part in development and maturation. Moreover, as proteins produced by these genes are detected in the cytoplasm of germ cells, the genes are thought to be possibly controlling the genesis of germ cells in concert with mitochondria. It would thus be suggested that mitochondria being the sole extra-nuclear organelle that possesses DNA may be involved in the gametogenesis, and this is yet to be investigated.

\section{DAZ family}

$D A Z$ is generally recognized to be localized at $A Z F C$ of the $Y$ chromosome, and to have a bearing on meiosis in the spermatogenesis. 
Approximately $80 \%$ of microdeletions in the $\mathrm{Y}$ chromosome are confined to this region. DAZLA and BOULE genes homologous to DAZ are localized on chromosomes 3 and 2, respectively, and both are involved not only in spermatogenesis but in oogenesis as well (Fig. 1). The hypothesis of gene expression of the $D A Z$ family in spermatogenesis suggested by Reijo et al. [2] is shown in Figure 2. Expression of the DAZLA gene is involved, like $D A Z$, in the entire process of germ cell development, taking part in the genesis of primordial germ cells and in the ensuing differentiation and maturation of those cells. Expression of the BOULE gene, on the other hand, culminates during the stage of meiosis. The $D A Z$ family is generally thought to be controlling RNA linkage. When the DAZLA gene expression was followed with the real-time PCR technique (Fig. 3), it became demonstrable also in the ovary with a delay about 35 cycles later than in the testis. Analysis of the fusion curve showed mutual concordance of ovarian and testicular gene amplification products. According to quantitative analysis of data on ovarian DAZLA gene expression by real-time $\mathrm{PCR}$ in age-stratified groups of subjects, there was no expression of this gene in a 72-year-old woman, suggesting an aging-related difference in the quantity of this gene expression.

\section{SYCP3}

This was reported as a spermatogenesis-related gene by Miyamoto et al. in 2004 [6]. Meiosis is the mode of cell division peculiar to germ cells, and has a bearing on the activity of synaptonemal complex (SC). In view of the fact that SYCP3 gene knockout leads to cessation of meiosis in mouse testis and gives rise to azoospermia in mice, Miyamoto et al. [6] performed a similar study in humans. Figure 4 illustrates the gene for SYCP3 and its amino acid sequence. A point mutation (643delA) identified in the study is shown in Figure 5 . This point mutation was localized heterologously, so that it
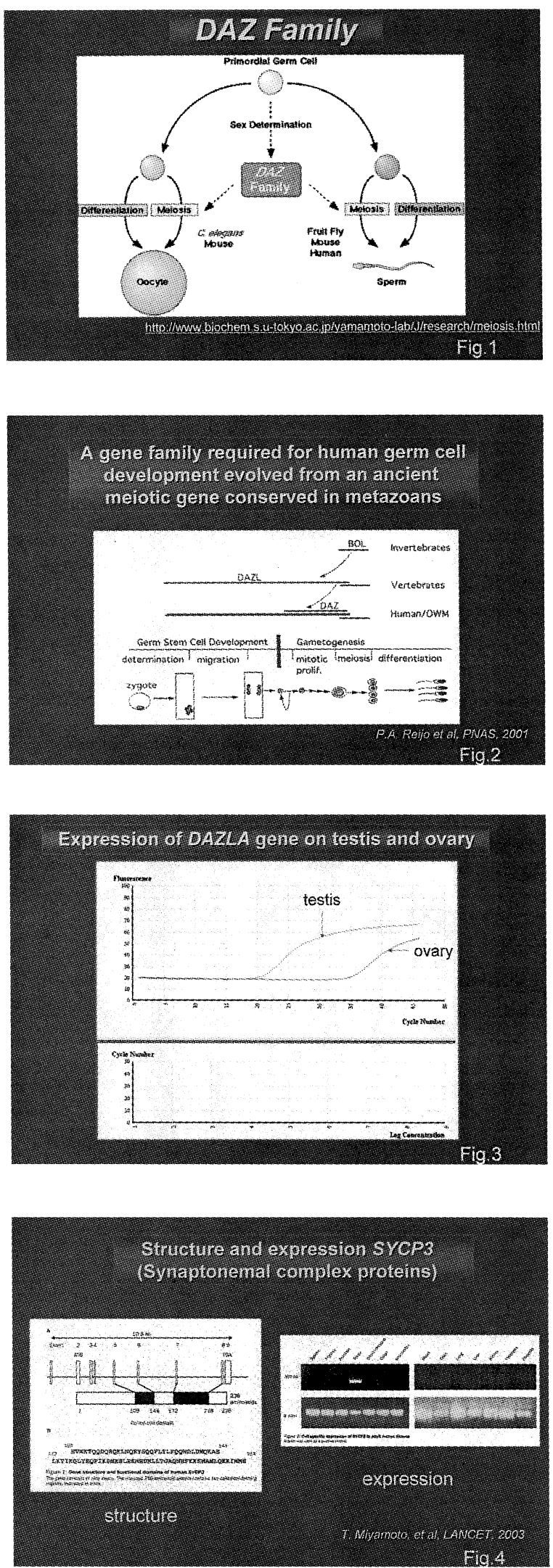


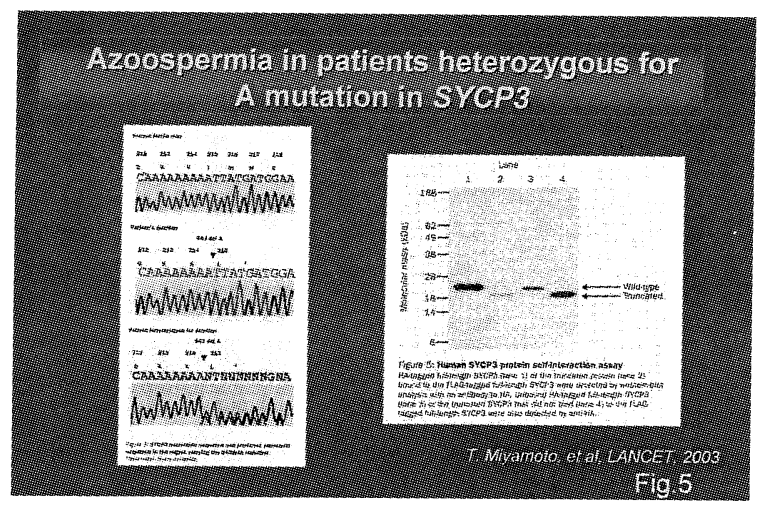

was considered to have caused formation of a heterogenous protein, giving rise to dysspermatogenesis. They have concluded that SYCP3, unlike the $D A Z$ family, is encoding DNA-binding protein.

\section{Summary}

Since the outset of investigation on the relationship between genes with gametogenesis, analyses have been made primarily of sex chromosomes. Studies of the $Y$ chromosome microdeletions in male infertility have progressed through analyses by many investigators and, as a result, their potential transmission to the following generation has come to pose a problem. Not only such genes on sex chromosomes as the $D A Z$ family and SYCP3 genes, but autosomal gene abnormalities should also be taken into account. These are extremely important to reproductive/hereditary counseling for couples wishing offspring (Fig. 6).

\section{Reference}

1) Tipolo L, Zuffardi O. 1976. Localization of Factors Controlling Spermatogenesis in the Nonfluorescent Portion of the Human $Y$ Chromosome Long arm. Hum Genet 34: 119-124.

2) Reijo R, Lee TY, Salo $P$, Alagappan $R$,

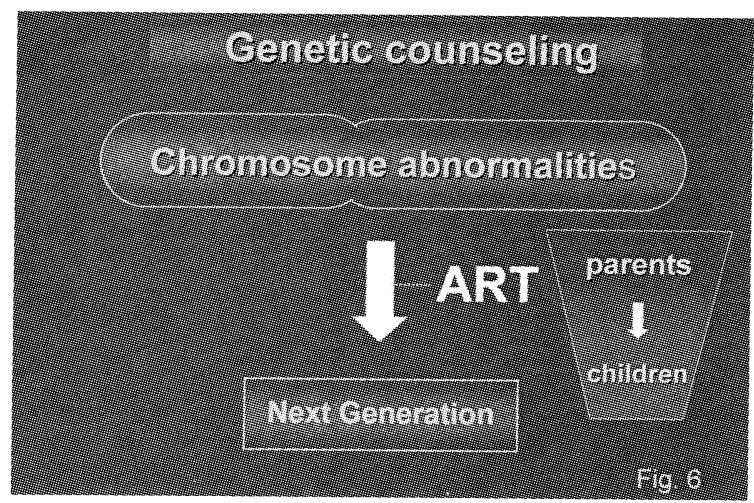

Brown LG, Rosenberg M, Rozen S, Jaffe T, Straus D, Hovatta O, Chapelle ADL, Silber S, Page DC. 1995. Diverse spermatogenic defects in humans caused by $Y$ chromosome deletions encompassing a novel RNA-binding protein gene. Nature Genetics 10: 383-384.

3) Kent-First MG, Muallen A, Shultz J, Pryor J, Roberts K, Nolten W, Meisner L, Chandley A, Gouchy G, Jorgensen L, Havighurst T, Grosch J. 1999. Defining Regions of the Y-Chromosome Responsible for Male Infertility and Identification of a Fourth AZF Region (AZFd) by Y-Chromosome Microdeletion Detection. Mol Reprod Development 53: 27-41.

4) Palermo G, Joris $H$, Devroey $P$, Van Steirteghem AC. 1992. Pregnancies after intracytoplasmic injection of single spermatozoon into an oocyte. Lancet 340 : 17-18.

5) Lin YM, ChenCW, Sun HS, Tsai SJ, Hsu CC, Teng YN, Lin JS, Kuo PL.. 2001. Expression patterns and transcriprt concentrations of the autosomal DAZL gene in the testes of azoospermic men. Mol Hum Reprod 7: 1015-1022,

6) Miyamoto $T$, Hasuike $S$, Yogev L, Maduro MR, Ishikawa M, Westphal H, Lamb DJ. 2003. Azoospermia in patients heterozygous for a mutation in SYCP3. Lancet 362: 1714-1719. 